L. da Silva, M. Spite, J. R. de Medeiros, eds.

\title{
In-Orbit Performance of the Far Ultraviolet Spectroscopic Explorer
}

\author{
G. Sonneborn
}

NASA/GSFC, Code 681, Greenbelt MD 20771 USA

H. W. Moos and K. R. Sembach

Dept. of Physics \& Astronomy, Johns Hopkins Univ., Baltimore, MD 21218 USA

The FUSE Science Team

\begin{abstract}
The Far Ultraviolet Spectroscopic Explorer (FUSE) satellite was launched by a Delta II rocket on 24 June 1999 into a $\sim 768 \mathrm{~km}, 25^{\circ}$ orbit. Following the In-Orbit Checkout phase, FUSE is obtaining highresolution spectra $(\lambda / \Delta \lambda \gtrsim 20,000)$ in the spectral range $905-1187 \AA$. The sensitivity meets or exceeds the pre-launch predictions. Many QSOs and AGNs have already been observed.
\end{abstract}

\section{FUSE In-Orbit Performance}

The FUSE satellite is fully operational and has been obtaining scientific data since 1999 Sept. Normal science operations for PI Team and Guest Investigator programs started on 1999 Dec. 1. Pointing performance is excellent $\left(<0.5^{\prime \prime}\right)$. The far-UV effective area meets or exceeds pre-flight predictions over the entire bandpass $\left(A_{\text {eff }}(\lambda) \sim 22 \mathrm{~cm}^{2}\right.$ to $\sim 70 \mathrm{~cm}^{2}$ when all four channels are combined). FUSE has four spectroscopic channels (pairs of mirrors and gratings), two coated with $\mathrm{SiC}$, two with $\mathrm{LiF}+\mathrm{Al}$. The background of the cross-delay-line microchannel-plate detectors are below the expected $1 \mathrm{ct} \mathrm{cm}^{-2} \mathrm{~s}^{-1}$. Scattered far-UV light in the instrument is extremely low. Several anomalies that effect observing efficiency, but not scientific data quality, have been encountered; several of these have been solved and remaining issues are being addressed. The grating and mirror positions in-orbit are not as stable as expected. Small alignment motions related to changing thermal environment of the satellite are primarily a function of solar orientation, but secondary thermal effects within a single orbit have also been observed. Nevertheless, scientific on-target observing efficiency was $25-35 \%$ in late 1999. Examples of FUSE spectra are shown in Fig. 1 and 2.

Spectral resolving power is currently (Jan. 2000) 20,000. Further improvements are expected as instrument optimization continues. For more information on all aspects of the FUSE mission, please consult the FUSE web site http://fuse.pha.jhu.edu. 


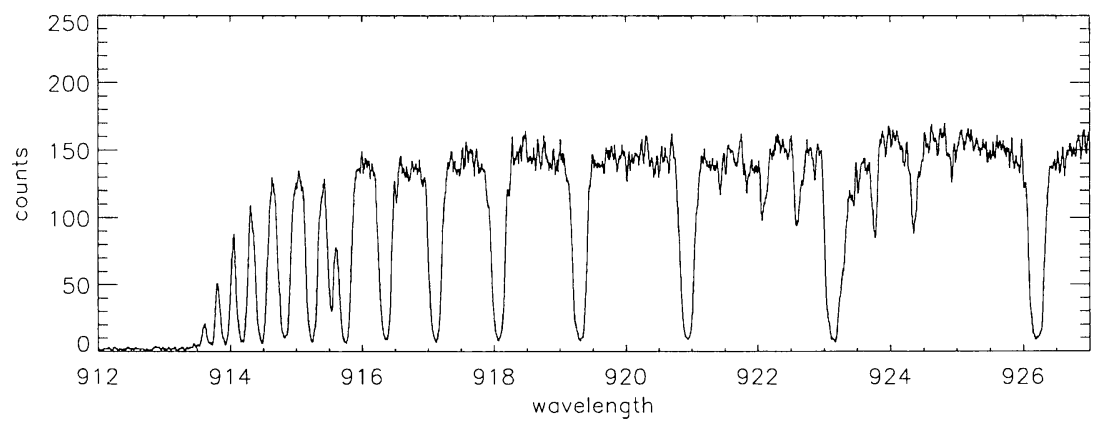

Figure 1. FUSE Commissioning Phase spectrum (resolution $\sim 20 \mathrm{~km}$ $\mathrm{s}^{-1}$ ) of the white dwarf WD2211-495 showing the interstellar H I Lyman

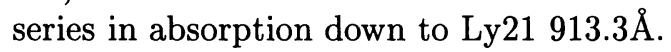
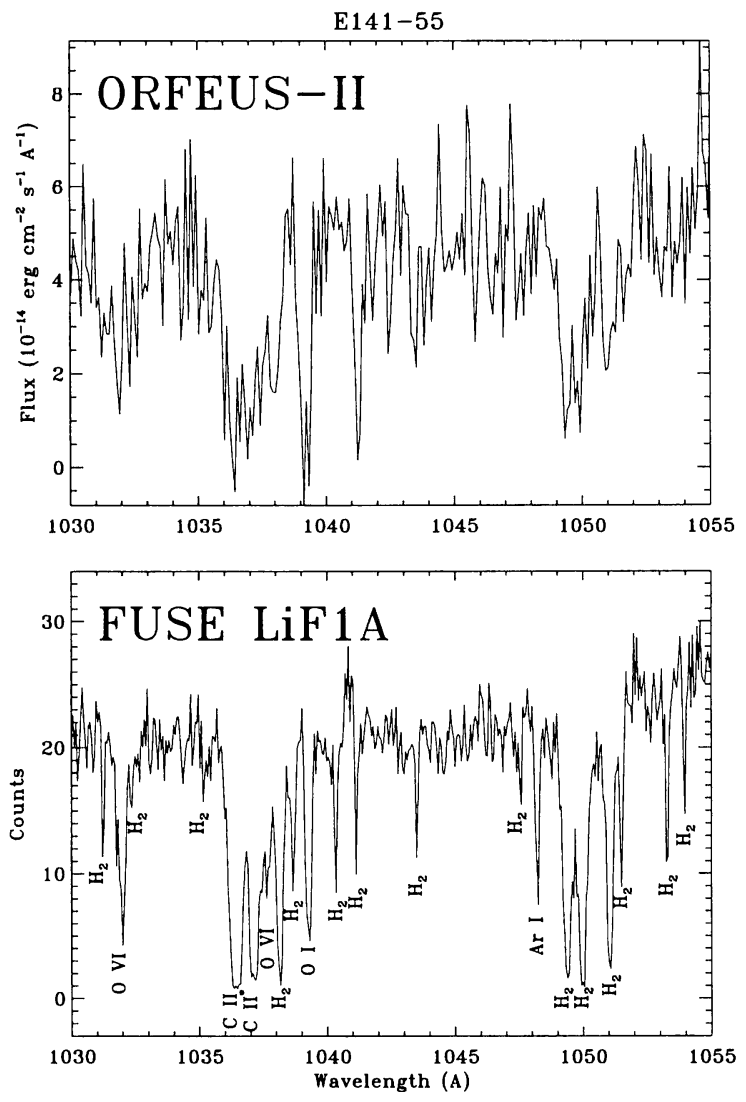

Figure 2. Comparison between FUSE and ORFEUS-II spectra of the Seyfert 1 galaxy ESO 141-55. The significant improvement in spectral resolution and sensitivity over previous missions is evident in the clarity with which the FUSE spectrum separates the $\mathrm{H}_{2}$ features from adjacent atomic lines. 


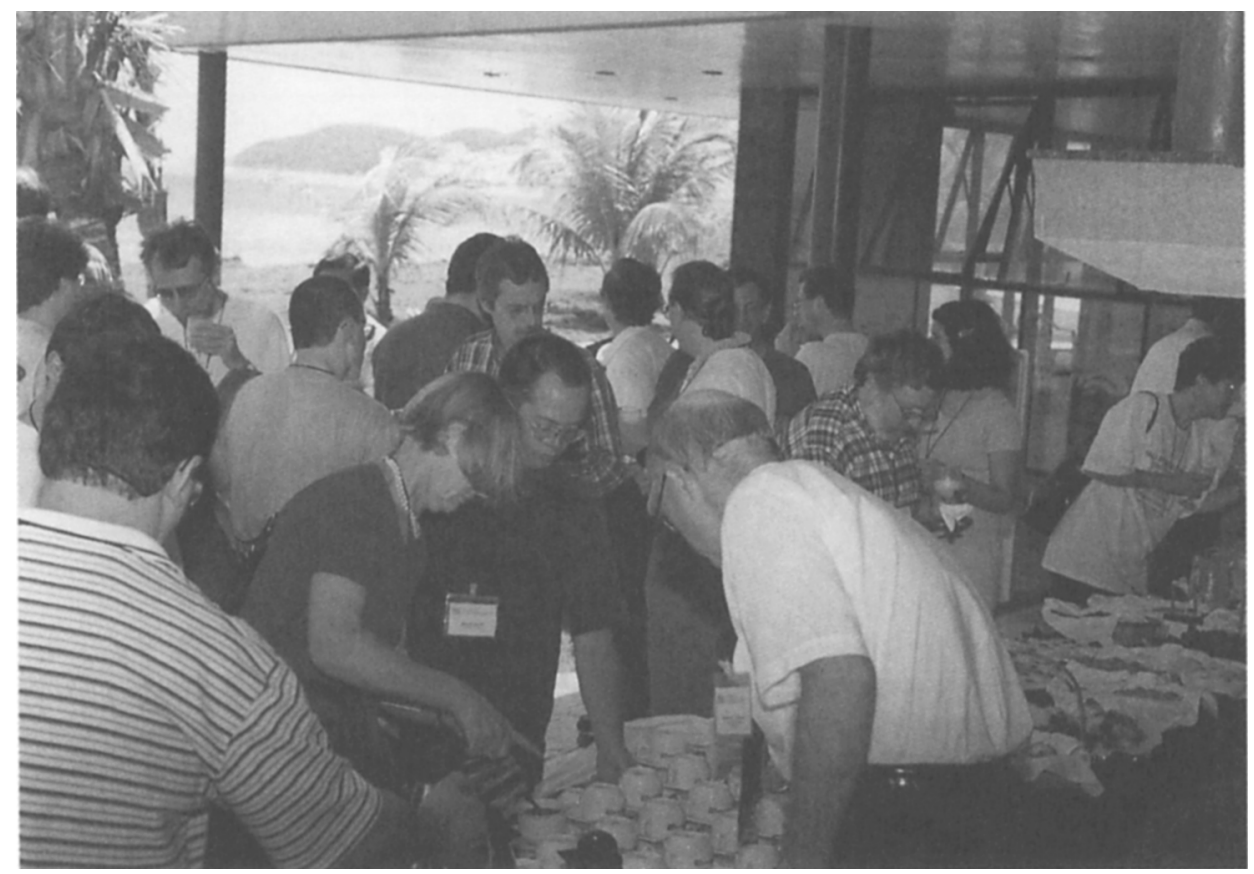

Coffee break 